Bull. Korean Math. Soc. 53 (2016), No. 1, pp. 61-81

http://dx.doi.org/10.4134/BKMS.2016.53.1.061

\title{
MONOTONE GENERALIZED CONTRACTIONS IN ORDERED METRIC SPACES
}

\author{
Aftab Alam and Mohammad Imdad
}

\begin{abstract}
In this paper, we prove some existence and uniqueness results on coincidence points for $g$-monotone mappings satisfying linear as well as generalized nonlinear contractivity conditions in ordered metric spaces. Our results generalize and extend two classical and well known results due to Ran and Reurings (Proc. Amer. Math. Soc. 132 (2004), no. 5, 14351443) and Nieto and Rodríguez-López (Acta Math. Sin. 23 (2007), no. 12, 2205-2212) besides similar other ones. Finally, as an application of one of our newly proved results, we establish the existence and uniqueness of solution of a first order periodic boundary value problem.
\end{abstract}

\section{Introduction}

The abstract monotone iterative techniques and corresponding fixed point results on ordered sets are natural as well as general enough to cover a variety of situations. There exists an extensive literature on this theme, but keeping in view the requirements of this presentation, we merely refer to ([4], [6], [9], [10], [11], [13], [14], [18], [25], [26], [38], [39], [40]). In recent years, a multitude of fixed point theorems have been proved in ordered metric spaces wherein the involved contraction conditions are merely assumed to hold on elements which are comparable in the underlying partial ordering. Thus, in this context, the usual contraction condition is considerably weakened but at the expense of monotonicity of the involved mapping. The techniques involved in the proofs of such results is the combination of ideas used in the proof of contraction principle together with the one involved in monotone iterative technique. This trend was essentially initiated by Turinici [39]. Later, Ran and Reurings [35] proved a slightly more natural version of the corresponding fixed point theorem of Turinici (cf. [39]) for continuous monotone mappings with some applications to matrix equations, which runs as follows.

Received December 11, 2014; Revised October 6, 2015.

2010 Mathematics Subject Classification. 47H10, 54H25.

Key words and phrases. ordered metric space, TCC property, termwise monotone sequence, c-bound. 
Theorem 1.1 (Ran and Reurings [35]). Let $(X, \preceq)$ be an ordered set equipped with a metric $d$ and $f$ a self-mapping on $X$. Suppose that the following conditions hold:

(i) $(X, d)$ is complete,

(ii) $f$ is monotone,

(iii) $f$ is continuous,

(iv) there exists $x_{0} \in X$ such that $x_{0} \preceq f\left(x_{0}\right)$ or $x_{0} \succeq f\left(x_{0}\right)$,

(v) there exists $\alpha \in[0,1)$ such that $d(f x, f y) \leq \alpha d(x, y) \quad \forall x, y \in X$ with $x \succeq y$,

(vi) every pair of elements of $X$ has a lower bound as well as an upper bound. Then $f$ has a unique fixed point.

Thereafter, Nieto and Rodríguez-López [32] slightly modified the assumptions (iii) and (vi) of Ran and Reurings' fixed point theorem and also given some applications to ordinary differential equations.

Theorem 1.2 (Nieto and Rodríguez-López [32]). Let $(X, \preceq)$ be an ordered set equipped with a metric $d$ and $f$ a self-mapping on $X$. Suppose that the following conditions hold:

(i) $(X, d)$ is complete,

(ii) $f$ is monotone,

(iii) either $f$ is continuous or $X$ satisfies the following property:

If $\left\{x_{n}\right\}$ is a sequence in $X$ such that $x_{n} \stackrel{d}{\longrightarrow} x$ whose consecutive terms are comparable, then there exists a subsequence $\left\{x_{n_{k}}\right\}$ of $\left\{x_{n}\right\}$ such that every term is comparable to the limit $x$,

(iv) there exists $x_{0} \in X$ such that $x_{0} \preceq f\left(x_{0}\right)$ or $x_{0} \succeq f\left(x_{0}\right)$,

(v) there exists $\alpha \in[0,1)$ such that $d(f x, f y) \leq \alpha d(x, y) \quad \forall x, y \in X$ with $x \succeq y$,

(vi) every pair of elements of $X$ has a lower bound or an upper bound.

Then $f$ has a unique fixed point.

In this continuation, Nieto and Rodríguez-López [31] proved some variants of Theorem 1.2 for increasing mappings, which has been generalized by many authors (e.g. [1], [2], [3], [5], [8], [12], [16], [17], [19], [20], [30], [33], [34], [41]) in the recent years. Most recently, Alam et al. [2] extended foregoing results to generalized $\varphi$-contractions.

The aim of this paper is to extend the core results of Ran and Reurings [35] (i.e., Theorem 1.1) and Nieto and Rodríguez-López [32] (i.e., Theorem 1.2) to a pair of mappings $f$ and $g$ defined on ordered metric space $X$ whenever $f$ is either $g$-monotone linear contraction or $g$-monotone nonlinear contraction in two different ways namely: $X$ is complete or alternately any subspace $Y$ of $X$ satisfying $f(X) \subseteq Y \subseteq g(X)$ is complete. This paper is a continuation of our earlier work carried out in [2]. 


\section{Preliminaries}

In this section, to make our exposition self-contained, we recall some basic definitions, relevant notions and auxiliary results. Throughout this paper, $\mathbb{N}$ stands for the set of natural numbers, while $\mathbb{N}_{0}$ for the set of whole numbers (i.e., $\left.\mathbb{N}_{0}=\mathbb{N} \cup\{0\}\right)$.

Definition 2.1 ([28]). A set $X$ together with a partial order $\preceq$ (often denoted by $(X, \preceq))$ is called an ordered set. In this context, we write $x \succeq y$ instead of $y \preceq x$. Two elements $x$ and $y$ in an ordered set $(X, \preceq)$ are said to be comparable if either $x \preceq y$ or $y \preceq x$ and we denote it as $x \prec \succ y$.

Clearly, the relation $\prec \succ$ is reflexive and symmetric, but not transitive in general.

Definition $2.2([33])$. A triplet $(X, d, \preceq)$ is called an ordered metric space if $(X, d)$ is a metric space and $(X, \preceq)$ is an ordered set.

Definition $2.3([12])$. Let $(X, \preceq)$ be an ordered set and $f$ and $g$ two selfmappings defined on $X$. We say that $f$ is $g$-increasing (resp. $g$-decreasing) if for any $x, y \in X g(x) \preceq g(y) \Rightarrow f(x) \preceq f(y)$ (resp.; $f(x) \succeq f(y))$. In all, $f$ is called $g$-monotone if $f$ is either $g$-increasing or $g$-decreasing.

Notice that under the restriction $g=I$, the identity mapping on $X$, the notions of $g$-increasing, $g$-decreasing and $g$-monotone mappings reduce to increasing, decreasing and monotone mappings respectively.

Proposition $2.1([2])$. Let $(X, \preceq)$ be an ordered set and $f$ and $g$ two selfmappings defined on $X$. If $f$ is $g$-monotone and $g(x)=g(y)$, then $f(x)=f(y)$.

Definition 2.4 ([22, 24]). Let $X$ be a nonempty set and $f$ and $g$ two selfmappings on $X$. Then

(i) an element $x \in X$ is called a coincidence point of $f$ and $g$ if

$$
g(x)=f(x),
$$

(ii) if $x \in X$ is a coincidence point of $f$ and $g$, then $\bar{x} \in X$ with $\bar{x}=g(x)=$ $f(x)$, is called a point of coincidence of $f$ and $g$

(iii) if $x \in X$ is a coincidence point of $f$ and $g$ such that $x=g(x)=f(x)$, then $x$ is called a common fixed point of $f$ and $g$,

(iv) the pair $(f, g)$ is said to be commuting if

$$
g(f x)=f(g x) \quad \forall x \in X \text { and }
$$

(v) the pair $(f, g)$ is said to be weakly compatible or coincidentally commuting if $f$ and $g$ commute at their coincidence points, i.e.,

$$
g(f x)=f(g x) \quad \text { whenever } g(x)=f(x) .
$$

Definition 2.5 ([23, 37]). Let $(X, d)$ be a metric space and $f$ and $g$ two selfmappings on $X$. Then 
(i) the pair $(f, g)$ is said to be weakly commuting if

$$
d(g f x, f g x) \leq d(g x, f x) \forall x \in X \text { and }
$$

(ii) the pair $(f, g)$ is said to be compatible if

$$
\lim _{n \rightarrow \infty} d\left(g f x_{n}, f g x_{n}\right)=0
$$

whenever $\left\{x_{n}\right\}$ is a sequence in $X$ such that

$$
\lim _{n \rightarrow \infty} g\left(x_{n}\right)=\lim _{n \rightarrow \infty} f\left(x_{n}\right) .
$$

It is clear that, in a metric space, commutativity $\Rightarrow$ weak commutativity $\Rightarrow$ compatibility $\Rightarrow$ weak compatibility but reverse implications are not true in general.

Definition 2.6 ([36]). Let $(X, d)$ be a metric space, $f$ and $g$ two self-mappings on $X$ and $x \in X$. We say that $f$ is $g$-continuous at $x$ if for all sequences $\left\{x_{n}\right\} \subset X$,

$$
g\left(x_{n}\right) \stackrel{d}{\longrightarrow} g(x) \Rightarrow f\left(x_{n}\right) \stackrel{d}{\longrightarrow} f(x) .
$$

Moreover, $f$ is called $g$-continuous if it is $g$-continuous at each point of $X$.

Notice that with $g=I$ (the identity mapping on $X$ ) Definition 2.6 reduces to the definition of continuity.

Now, we formulate the variants of bounded sequences and monotone sequences with respect to relation $\prec \succ$.

Definition 2.7. Let $(X, \preceq)$ be an ordered set and $\left\{x_{n}\right\}$ a sequence in $X$. Then

(i) $\left\{x_{n}\right\}$ is said to be termwise bounded if there is an element $z \in X$ such that each term of $\left\{x_{n}\right\}$ is comparable with $z$, i.e.,

$$
x_{n} \prec \succ z \quad \forall n \in \mathbb{N}_{0}
$$

so that $z$ is a c-bound of $\left\{x_{n}\right\}$ and

(ii) $\left\{x_{n}\right\}$ is said to be termwise monotone if consecutive terms of $\left\{x_{n}\right\}$ are comparable, i.e.,

$$
x_{n} \prec \succ x_{n+1} \forall n \in \mathbb{N}_{0} .
$$

Clearly all bounded above as well as bounded below sequences are termwise bounded and all monotone sequences are termwise monotone.

Let $(X, d, \preceq)$ be an ordered metric space and $\left\{x_{n}\right\}$ a sequence in $X$. If $\left\{x_{n}\right\}$ is termwise monotone and $x_{n} \stackrel{d}{\longrightarrow} x$, then we denote it symbolically by $x_{n} \uparrow x$.

Next, we formulate the following notion using certain property utilized by Nieto and Rodríguez-López [32] (see assumption (iii) in Theorem 1.2).

Definition 2.8. Let $(X, d, \preceq)$ be an ordered metric space. We say that $(X, d$, $\preceq$ ) has $T C C$ (termwise monotone-convergence-c-bound) property if every termwise monotone convergent sequence $\left\{x_{n}\right\}$ in $X$ has a subsequence, which is termwise bounded by the limit (of the sequence) as a c-bound, i.e.,

$$
x_{n} \uparrow x \Rightarrow \exists \text { a subsequence }\left\{x_{n_{k}}\right\} \text { of }\left\{x_{n}\right\} \text { with } x_{n_{k}} \prec \succ x \forall k \in \mathbb{N}_{0} \text {. }
$$


Definition 2.9. Let $(X, d, \preceq)$ be an ordered metric space and $g$ a self-mapping on $X$. We say that $(X, d, \preceq)$ has $g$-TCC property if every termwise monotone convergent sequence $\left\{x_{n}\right\}$ in $X$ has a subsequence, whose $g$-image is termwise bounded by $g$-image of limit (of the sequence) as a c-bound, i.e.,

$x_{n} \uparrow x \Rightarrow \exists$ a subsequence $\left\{x_{n_{k}}\right\}$ of $\left\{x_{n}\right\}$ with $g\left(x_{n_{k}}\right) \prec \succ g(x) \forall k \in \mathbb{N}_{0}$.

Notice that under the restriction $g=I$, the identity mapping on $X$, Definition 2.9 reduces to Definition 2.8 .

Definition 2.10. An ordered set $(X, \preceq)$ is called sequentially chainable if range of every termwise monotone sequence in $X$ remains a totally ordered subset of $X$.

Proposition 2.2. The following are equivalent:

(i) $(X, \preceq)$ is sequentially chainable,

(ii) $\prec \succ$ is transitive on range of every termwise monotone sequence in $X$,

(iii) for every termwise monotone sequence $\left\{x_{n}\right\}$ in $X$,

$$
x_{n} \prec \succ x_{m} \forall n, m \in \mathbb{N}_{0} .
$$

The following family of control functions is essentially due to Boyd and Wong $[7]$.

$$
\begin{aligned}
\Psi=\{\varphi:[0, \infty) \rightarrow[0, \infty): & \varphi(t)<t \text { for each } t>0 \text { and } \\
& \varphi \text { is right-upper semicontinuous }\} .
\end{aligned}
$$

Mukherjea [29] introduced the following family of control functions:

$\Theta=\{\varphi:[0, \infty) \rightarrow[0, \infty): \varphi(t)<t$ for each $t>0$ and $\varphi$ is right continuous $\}$.

The following family of control functions found in literature is more natural.

$$
\Im=\{\varphi:[0, \infty) \rightarrow[0, \infty): \varphi(t)<t \text { for each } t>0 \text { and } \varphi \text { is continuous }\} .
$$

The following family of control functions is due to Lakshmikantham and Cirić $[27]$.

$$
\begin{array}{r}
\Phi=\{\varphi:[0, \infty) \rightarrow[0, \infty): \varphi(t)<t \text { for each } t>0 \text { and } \\
\left.\lim _{r \rightarrow t^{+}} \varphi(r)<t \text { for each } t>0\right\} .
\end{array}
$$

The following family of control functions is indicated in Boyd and Wong [7] but was later used in Jotic [21].

$$
\begin{aligned}
\Omega=\{\varphi:[0, \infty) \rightarrow[0, \infty): & \varphi(t)<t \text { for each } t>0 \text { and } \\
& \underset{r \rightarrow t^{+}}{\limsup \varphi(r)<t \text { for each } t>0\} .}
\end{aligned}
$$


Proposition $2.3([2])$. The class $\Omega$ enlarges the classes $\Psi, \Theta, \Im$ and $\Phi$ under the following inclusion relation:

$$
\Im \subset \Theta \subset \Psi \subset \Omega \text { and } \Im \subset \Theta \subset \Phi \subset \Omega .
$$

The following known results are useful in the proof of our main results.

Lemma $2.1([2])$. Let $\varphi \in \Omega$. If $\left\{a_{n}\right\} \subset(0, \infty)$ is a sequence such that $a_{n+1} \leq \varphi\left(a_{n}\right) \forall n \in \mathbb{N}_{0}$, then $\lim _{n \rightarrow \infty} a_{n}=0$.

Lemma 2.2 ([20]). Let $(X, d)$ be a metric space and $\left\{x_{n}\right\}$ a sequence in $X$ such that $\lim _{n \rightarrow \infty} d\left(x_{n}, x_{n+1}\right)=0$. If $\left\{x_{n}\right\}$ is not a Cauchy sequence, then there exist $\epsilon>0$ and two subsequences $\left\{x_{n_{k}}\right\}$ and $\left\{x_{m_{k}}\right\}$ of $\left\{x_{n}\right\}$ such that

(i) $n_{k}>m_{k} \geq k$,

(ii) $d\left(x_{m_{k}}, x_{n_{k}}\right) \geq \epsilon$,

(iii) $d\left(x_{m_{k}}, x_{n_{k}-1}\right)<\epsilon$,

(iv) the following four sequences tend to $\epsilon$ when $k \rightarrow \infty$ :

$$
d\left(x_{m_{k}}, x_{n_{k}}\right), d\left(x_{m_{k}+1}, x_{n_{k}}\right), d\left(x_{m_{k}}, x_{n_{k}+1}\right), d\left(x_{m_{k}+1}, x_{n_{k}+1}\right) .
$$

Lemma 2.3 ([15]). Let $X$ be a nonempty set and $g$ a self-mapping on $X$. Then there exists a subset $E \subseteq X$ such that $g(E)=g(X)$ and $g: E \rightarrow X$ is one-one.

\section{Results on coincidence points}

Firstly, we prove a coincidence point theorem under generalized $\varphi$-contractivity condition as follows.

Theorem 3.1. Let $(X, d, \preceq)$ be an ordered metric space and $f$ and $g$ two selfmappings on $X$. Suppose that the following conditions hold:

(a) $f(X) \subseteq g(X)$,

(b) $f$ is g-monotone,

(c) there exists $x_{0} \in X$ such that $g\left(x_{0}\right) \prec \succ f\left(x_{0}\right)$,

(d) there exists $\varphi \in \Omega$ such that $d(f x, f y) \leq \varphi(d(g x, g y)) \forall x, y \in X$ with $g(x) \prec \succ g(y)$

(e) (e1) $(X, d)$ is complete,

(e2) $(f, g)$ is compatible pair,

(e3) $g$ is continuous,

(e4) either $f$ is continuous or $(X, d, \preceq)$ has g-TCC property,

or alternately

$\left(\mathrm{e}^{\prime}\right)\left(\mathrm{e}^{\prime} 1\right)$ there exists a subset $Y$ of $X$ such that $f(X) \subseteq Y \subseteq g(X)$ and $(Y, d)$ is complete,

$\left(\mathrm{e}^{\prime} 2\right)$ either $f$ is $g$-continuous or $f$ and $g$ are continuous or $(Y, d, \preceq)$ has TCC property,

(f) $(f X, \preceq)$ is sequentially chainable.

Then $f$ and $g$ have a coincidence point.

Proof. In view of assumption (c) if $g\left(x_{0}\right)=f\left(x_{0}\right)$, then $x_{0}$ is a coincidence point of $f$ and $g$ and hence we are through. Otherwise, if $g\left(x_{0}\right) \neq f\left(x_{0}\right)$, 
then owing to assumption (a) (i.e., $f(X) \subseteq g(X)$ ), we can choose $x_{1} \in X$ such that $g\left(x_{1}\right)=f\left(x_{0}\right)$. As $f(X) \subseteq g(X)$, we can choose $x_{2} \in X$ such that $g\left(x_{2}\right)=f\left(x_{1}\right)$. Continuing this process, we define a sequence $\left\{x_{n}\right\} \subset X$ of joint iterates such that

$$
g\left(x_{n+1}\right)=f\left(x_{n}\right) \quad \forall n \in \mathbb{N}_{0} .
$$

Now, we show that $\left\{g x_{n}\right\}$ is a termwise monotone sequence, i.e.,

$$
g\left(x_{n}\right) \prec \succ g\left(x_{n+1}\right) \forall n \in \mathbb{N}_{0}
$$

To prove (2), we distinguish four cases (owing to conditions (b) and (c):

(i) $f$ is $g$-increasing and $g\left(x_{0}\right) \preceq f\left(x_{0}\right)$,

(ii) $f$ is $g$-increasing and $g\left(x_{0}\right) \succeq f\left(x_{0}\right)$,

(iii) $f$ is $g$-decreasing and $g\left(x_{0}\right) \preceq f\left(x_{0}\right)$,

(iv) $f$ is $g$-decreasing and $g\left(x_{0}\right) \succeq f\left(x_{0}\right)$.

In cases (i) and (ii), we conclude that $\left\{g x_{n}\right\}$ is respectively increasing and decreasing sequence (for proof see lines of the main results of [2]).

In case (iii), using (1) (with $n=0$ ), we have $g\left(x_{0}\right) \preceq f\left(x_{0}\right)=g\left(x_{1}\right)$. Hence, on using assumption (b) and (1), we get $g\left(x_{1}\right)=f\left(x_{0}\right) \succeq f\left(x_{1}\right)=g\left(x_{2}\right)$. Further, on using assumption (b) and (1), we get $g\left(x_{2}\right)=f\left(x_{1}\right) \preceq f\left(x_{2}\right)=$ $g\left(x_{3}\right)$. Continuing this procedure inductively, we obtain

$$
g\left(x_{0}\right) \preceq g\left(x_{1}\right), g\left(x_{1}\right) \succeq g\left(x_{2}\right), g\left(x_{2}\right) \preceq g\left(x_{3}\right), \ldots
$$

In the similar manner, in case (iv), we obtain

$$
g\left(x_{0}\right) \succeq g\left(x_{1}\right), g\left(x_{1}\right) \preceq g\left(x_{2}\right), g\left(x_{2}\right) \succeq g\left(x_{3}\right), \ldots
$$

Therefore, in all the cases, (2) holds for all $n \in \mathbb{N}_{0}$.

If $g\left(x_{n_{0}}\right)=g\left(x_{n_{0}+1}\right)$ for some $n_{0} \in \mathbb{N}$, then using (1), we have $g\left(x_{n_{0}}\right)=$ $f\left(x_{n_{0}}\right)$, i.e., $x_{n_{0}}$ is a coincidence point of $f$ and $g$ so that we are through. On the other hand, if $g\left(x_{n}\right) \neq g\left(x_{n+1}\right)$ for each $n \in \mathbb{N}_{0}$, we define a sequence $\left\{d_{n}\right\}_{n=0}^{\infty} \subset(0, \infty)$, where

$$
d_{n}:=d\left(g x_{n}, g x_{n+1}\right) .
$$

On using (1), (2), (3) and assumption (d), we obtain

$$
\begin{aligned}
d_{n+1} & =d\left(g x_{n+1}, g x_{n+2}\right) \\
& =d\left(f x_{n}, f x_{n+1}\right) \\
& \leq \varphi\left(d\left(g x_{n}, g x_{n+1}\right)\right) \\
& =\varphi\left(d_{n}\right)
\end{aligned}
$$

so that

$$
d_{n+1} \leq \varphi\left(d_{n}\right)
$$

Hence by Lemma 2.1, we obtain

$$
\lim _{n \rightarrow \infty} d_{n}=\lim _{n \rightarrow \infty} d\left(g x_{n}, g x_{n+1}\right)=0 .
$$


Now, in view of (4) and Lemma 2.2, there exist $\epsilon>0$ and two subsequences $\left\{g x_{n_{k}}\right\}$ and $\left\{g x_{m_{k}}\right\}$ of $\left\{g x_{n}\right\}$ such that $n_{k}>m_{k} \geq k, d\left(g x_{m_{k}}, g x_{n_{k}}\right) \geq$ $\epsilon, d\left(g x_{m_{k}}, g x_{n_{k}-1}\right)<\epsilon$ and

$$
\begin{aligned}
\lim _{k \rightarrow \infty} d\left(g x_{m_{k}}, g x_{n_{k}}\right) & =\lim _{k \rightarrow \infty} d\left(g x_{m_{k}+1}, g x_{n_{k}}\right) \\
& =\lim _{k \rightarrow \infty} d\left(g x_{m_{k}}, g x_{n_{k}+1}\right) \\
& =\lim _{k \rightarrow \infty} d\left(g x_{m_{k}+1}, g x_{n_{k}+1}\right)=\epsilon .
\end{aligned}
$$

Denote $r_{k}:=d\left(g x_{m_{k}}, g x_{n_{k}}\right)$. By (1), we have $\left\{g x_{n}\right\} \subset f(X)$. Due to (f), (2) and Proposition 2.2, we have $g\left(x_{m_{k}}\right) \prec \succ g\left(x_{n_{k}}\right)$. Hence, on using (1) and assumption (d), we obtain

$$
\begin{aligned}
d\left(g x_{m_{k}+1}, g x_{n_{k}+1}\right) & =d\left(f x_{m_{k}}, f x_{n_{k}}\right) \\
& \leq \varphi\left(d\left(g x_{m_{k}}, g x_{n_{k}}\right)\right) . \\
& =\varphi\left(r_{k}\right)
\end{aligned}
$$

so that

$$
d\left(g x_{m_{k}+1}, g x_{n_{k}+1}\right) \leq \varphi\left(r_{k}\right) .
$$

On taking limit superior as $k \rightarrow \infty$ in (6) and using (5) and the definition of $\Omega$, we have

$$
\epsilon=\limsup _{k \rightarrow \infty} d\left(g x_{m_{k}+1}, g x_{n_{k}+1}\right) \leq \limsup _{k \rightarrow \infty} \varphi\left(r_{k}\right)=\limsup _{r_{k} \rightarrow \epsilon^{+}} \varphi\left(r_{k}\right)<\epsilon,
$$

which is a contradiction. Therefore $\left\{g x_{n}\right\}$ is a Cauchy sequence. Now, we use assumptions (e) or ( $\left.\mathrm{e}^{\prime}\right)$ to accomplish the proof.

Firstly, assume that (e) holds. By assumption (e1) (i.e., the completeness of $X)$, there exists $z \in X$ such that

$$
\lim _{n \rightarrow \infty} g\left(x_{n}\right)=z .
$$

On using (1) and (7), we obtain

$$
\lim _{n \rightarrow \infty} f\left(x_{n}\right)=\lim _{n \rightarrow \infty} g\left(x_{n+1}\right)=z .
$$

In view of assumption (e3) (i.e., continuity of $g$ ) in (7) and (8), we have

$$
\begin{aligned}
& \lim _{n \rightarrow \infty} g\left(g x_{n}\right)=g\left(\lim _{n \rightarrow \infty} g x_{n}\right)=g(z), \\
& \lim _{n \rightarrow \infty} g\left(f x_{n}\right)=g\left(\lim _{n \rightarrow \infty} f x_{n}\right)=g(z) .
\end{aligned}
$$

As $\lim _{n \rightarrow \infty} f\left(x_{n}\right)=\lim _{n \rightarrow \infty} g\left(x_{n}\right)=z$ (due to (7) and (8)), on using assumption (e2) (i.e., compatibility of $f$ and $g$ ), we obtain

$$
\lim _{n \rightarrow \infty} d\left(g f x_{n}, f g x_{n}\right)=0 .
$$


Now, we show that $z$ is a coincidence point of $f$ and $g$. To accomplish this, we use assumption (e4). Suppose that $f$ is continuous. On using (7) and continuity of $f$, we obtain

$$
\lim _{n \rightarrow \infty} f\left(g x_{n}\right)=f\left(\lim _{n \rightarrow \infty} g x_{n}\right)=f(z) .
$$

On using (10), (11), (12) and continuity of $d$, we obtain

$$
\begin{aligned}
d(g z, f z) & =d\left(\lim _{n \rightarrow \infty} g f x_{n}, \lim _{n \rightarrow \infty} f g x_{n}\right) \\
& =\lim _{n \rightarrow \infty} d\left(g f x_{n}, f g x_{n}\right)=0
\end{aligned}
$$

so that

$$
g(z)=f(z)
$$

Thus $z \in X$ is a coincidence point of $f$ and $g$ and hence we are through.

Alternately, suppose that $\left(X, d, \preceq\right.$ ) has $g$-TCC property. As $g\left(x_{n}\right) \uparrow z$ (due to $(2)$ and (7)), $\exists$ a subsequence $\left\{y_{n_{k}}\right\}$ of $\left\{g x_{n}\right\}$ such that

$$
g\left(y_{n_{k}}\right) \prec \succ g(z) \forall k \in \mathbb{N}_{0} .
$$

Now $\left\{g x_{n}\right\} \subset g(X)$ and $\left\{y_{n_{k}}\right\} \subset\left\{g x_{n}\right\}, \exists\left\{x_{n_{k}}\right\} \subset X$ such that $y_{n_{k}}=g\left(x_{n_{k}}\right)$

$$
\Longrightarrow \quad g\left(g x_{n_{k}}\right) \prec \succ g(z) \forall k \in \mathbb{N}_{0} \text {. }
$$

Since $g\left(x_{n_{k}}\right) \stackrel{d}{\longrightarrow} z$, so equations (7)-(12) also hold for $\left\{x_{n_{k}}\right\}$ instead of $\left\{x_{n}\right\}$. On using (13) and assumption $(d)$, we obtain

$$
d\left(f g x_{n_{k}}, f z\right) \leq \varphi\left(d\left(g g x_{n_{k}}, g z\right)\right) \forall k \in \mathbb{N}_{0} .
$$

Now, we asserts that

$$
d\left(f g x_{n_{k}}, f z\right) \leq d\left(g g x_{n_{k}}, g z\right) \forall k \in \mathbb{N} .
$$

On account of two different possibilities arising here, we consider a partition $\left\{\mathbb{N}^{0}, \mathbb{N}^{+}\right\}$of $\mathbb{N}$, i.e., $\mathbb{N}^{0} \cup \mathbb{N}^{+}=\mathbb{N}$ and $\mathbb{N}^{0} \cap \mathbb{N}^{+}=\emptyset$ verifying that

(i) $d\left(g g x_{n_{k}}, g z\right)=0 \quad \forall k \in \mathbb{N}^{0}$,

(ii) $d\left(g g x_{n_{k}}, g z\right)>0 \quad \forall k \in \mathbb{N}^{+}$.

In case (i), on using Proposition 2.1, we get $d\left(f g x_{n_{k}}, f z\right)=0 \forall k \in \mathbb{N}^{0}$ and hence (14) holds for all $k \in \mathbb{N}^{0}$. In case (ii), owing to the definition of $\Omega$, we have $d\left(f g x_{n_{k}}, f z\right) \leq \varphi\left(d\left(g g x_{n_{k}}, g z\right)\right)<d\left(g g x_{n_{k}}, g z\right) \forall k \in \mathbb{N}^{+}$and hence (14) holds for all $k \in \mathbb{N}^{+}$. Thus (14) holds for all $k \in \mathbb{N}$.

On using triangular inequality, (9), (10), (11) and (14), we get

$$
\begin{aligned}
d(g z, f z) & \leq d\left(g z, g f x_{n_{k}}\right)+d\left(g f x_{n_{k}}, f g x_{n_{k}}\right)+d\left(f g x_{n_{k}}, f z\right) \\
& \leq d\left(g z, g f x_{n_{k}}\right)+d\left(g f x_{n_{k}}, f g x_{n_{k}}\right)+d\left(g g x_{n_{k}}, g z\right) \\
& \rightarrow 0 \text { as } k \rightarrow \infty
\end{aligned}
$$

so that

$$
g(z)=f(z)
$$

Thus $z \in X$ is a coincidence point of $f$ and $g$ and hence we are through. 
Now, assume that $\left(\mathrm{e}^{\prime}\right)$ holds. Then the assumption $f(X) \subseteq Y$ and completeness of $Y$ ensure the existence of $y \in Y$ such that $f\left(x_{n}\right) \stackrel{d}{\longrightarrow} y$. Again owing to assumption $Y \subseteq g(X)$, we can find $u \in X$ such that $y=g(u)$. Hence, on using (1), we get

$$
\lim _{n \rightarrow \infty} g\left(x_{n}\right)=\lim _{n \rightarrow \infty} f\left(x_{n}\right)=g(u) .
$$

Now, we show that $u$ is a coincidence point of $f$ and $g$. To accomplish this, we use assumption $\left(\mathrm{e}^{\prime} 2\right)$. Firstly, suppose that $f$ is $g$-continuous, then using (15), we get

$$
\lim _{n \rightarrow \infty} f\left(x_{n}\right)=f(u) .
$$

On using (15) and (16), we get

$$
g(u)=f(u) .
$$

Secondly, suppose that $f$ and $g$ are continuous. Owing to Lemma 2.3, there exists a subset $E \subseteq X$ such that $g(E)=g(X)$ and $g: E \rightarrow X$ is one-one. Without loss of generality, we are able to choose $E \subseteq X$ such that $u \in E$. Now, define $T: g(E) \rightarrow g(X)$ by

$$
T(g e)=f(e) \forall g(e) \in g(E) \text { where } e \in E .
$$

As $g: E \rightarrow X$ is one-one and $f(X) \subseteq g(X), T$ is well defined. Again, as $f$ and $g$ are continuous, it follows that $T$ is continuous. Since $\left\{x_{n}\right\} \subset X$ and $g(E)=g(X)$, there exists $\left\{e_{n}\right\} \subset E$ such that $g\left(x_{n}\right)=g\left(e_{n}\right) \forall n \in \mathbb{N}_{0}$. On using Proposition 2.1, we get $f\left(x_{n}\right)=f\left(e_{n}\right) \forall n \in \mathbb{N}_{0}$. Therefore, in view of (1) and (15), we get

$$
\lim _{n \rightarrow \infty} g\left(e_{n}\right)=\lim _{n \rightarrow \infty} f\left(e_{n}\right)=g(u) .
$$

On using (17), (18) and continuity of $T$, we get

$$
f(u)=T(g u)=T\left(\lim _{n \rightarrow \infty} g e_{n}\right)=\lim _{n \rightarrow \infty} T\left(g e_{n}\right)=\lim _{n \rightarrow \infty} f\left(e_{n}\right)=g(u) .
$$

Thus $u \in X$ is a coincidence point of $f$ and $g$ and hence we are through.

Finally, suppose that $(Y, d, \preceq)$ has $T C C$ property. As $g\left(x_{n}\right) \uparrow g(u)$ (due to (2) and (15)), $\exists$ a subsequence $\left\{g x_{n_{k}}\right\}$ of $\left\{g x_{n}\right\}$ such that

$$
g\left(x_{n_{k}}\right) \prec \succ g(u) \forall k \in \mathbb{N}_{0} .
$$

On using (1), (19) and assumption (d), we obtain

$$
d\left(g x_{n_{k}+1}, f u\right)=d\left(f x_{n_{k}}, f u\right) \leq \varphi\left(d\left(g x_{n_{k}}, g u\right)\right) \forall k \in \mathbb{N}_{0} .
$$

We asserts that

$$
d\left(g x_{n_{k}+1}, f u\right) \leq d\left(g x_{n_{k}}, g u\right) \forall k \in \mathbb{N} .
$$

On account of two different possibilities arising here, we consider a partition $\left\{\mathbb{N}^{0}, \mathbb{N}^{+}\right\}$of $\mathbb{N}$, i.e., $\mathbb{N}^{0} \cup \mathbb{N}^{+}=\mathbb{N}$ and $\mathbb{N}^{0} \cap \mathbb{N}^{+}=\emptyset$ verifying that

(i) $d\left(g x_{n_{k}}, g u\right)=0 \quad \forall k \in \mathbb{N}^{0}$,

(ii) $d\left(g x_{n_{k}}, g u\right)>0 \quad \forall k \in \mathbb{N}^{+}$. 
In case (i) holds, on using Proposition 2.1, we get $d\left(f x_{n_{k}}, f u\right)=0 \forall k \in$ $\mathbb{N}^{0}$, which implies that $d\left(g x_{n_{k}+1}, f u\right)=0 \forall k \in \mathbb{N}^{0}$ and hence (20) holds for all $k \in \mathbb{N}^{0}$. If case (ii) holds, then owing to the definition of $\Omega$, we have $d\left(g x_{n_{k}+1}, f u\right) \leq \varphi\left(d\left(g x_{n_{k}}, g u\right)\right)<d\left(g x_{n_{k}}, g u\right) \forall k \in \mathbb{N}^{+}$and hence $(20)$ holds for all $k \in \mathbb{N}^{+}$. Thus (20) holds for all $k \in \mathbb{N}$.

On using (15), (20) and continuity of $d$, we get

$$
\begin{aligned}
d(g u, f u) & =d\left(\lim _{k \rightarrow \infty} g x_{n_{k}+1}, f u\right) \\
& =\lim _{k \rightarrow \infty} d\left(g x_{n_{k}+1}, f u\right) \\
& \leq \lim _{k \rightarrow \infty} d\left(g x_{n_{k}}, g u\right)=0
\end{aligned}
$$

so that

$$
g(u)=f(u)
$$

Hence $u \in X$ is a coincidence point of $f$ and $g$. This completes the proof.

Remark 3.1. In view of Proposition 2.3, Theorem 3.1 remains true if we replace the class $\Omega$ by anyone of the classes $\Psi, \Theta, \Im$ and $\Phi$.

If we set $\varphi(t)=\alpha t$ (with $\alpha \in[0,1))$ in Theorem 3.1 and remove the assumption (f), then we obtain the following coincidence theorem for $\alpha$-contraction.

Theorem 3.2. Let $(X, d, \preceq)$ be an ordered metric space and $f$ and $g$ two selfmappings on $X$. Suppose that the following conditions hold:

(a) $f(X) \subseteq g(X)$,

(b) $f$ is g-monotone,

(c) there exists $x_{0} \in X$ such that $g\left(x_{0}\right) \prec \succ f\left(x_{0}\right)$,

(d) there exists $\alpha \in[0,1)$ such that $d(f x, f y) \leq \alpha d(g x, g y) \forall x, y \in X$ with $g(x) \prec \succ g(y)$

(e) (e1) $(X, d)$ is complete,

(e2) $(f, g)$ is compatible pair,

(e3) $g$ is continuous,

(e4) either $f$ is continuous or $(X, d, \preceq)$ has g-TCC property,

or alternately

$\left(\mathrm{e}^{\prime}\right)\left(\mathrm{e}^{\prime} 1\right)$ there exists a subset $Y$ of $X$ such that $f(X) \subseteq Y \subseteq g(X)$ and $(Y, d)$ is complete,

$\left(\mathrm{e}^{\prime} 2\right)$ either $f$ is $g$-continuous or $f$ and $g$ are continuous or $(Y, d, \preceq)$ has TCC property.

Then $f$ and $g$ have a coincidence point.

Proof. We use the same structure as in the proof of Theorem 3.1. By following its lines, we derive

$$
d\left(g x_{n+1}, g x_{n+2}\right)=d\left(f x_{n}, f x_{n+1}\right) \leq \alpha d\left(g x_{n}, g x_{n+1}\right) \forall n \in \mathbb{N}_{0},
$$

it follows that,

$$
d\left(g x_{n}, g x_{n+1}\right) \leq \alpha^{n} d\left(g x_{0}, g x_{1}\right) \forall n \in \mathbb{N}_{0} .
$$


By classical techniques, it can be easily shown that $\left\{g x_{n}\right\}$ is a Cauchy sequence. Here it is noticed that there is no need to use the assumption (f) mentioned in Theorem 3.1, because we do not need to apply the contractivity condition to $d\left(g x_{m_{k}}, g x_{n_{k}}\right)$. Finally, we accomplish the proof by using (e) and ( $\left.\mathrm{e}^{\prime}\right)$ same as in the proof of Theorem 3.1 .

Corollary 3.1. Theorem 3.1 (also Theorem 3.2) remains true if we replace $\left(e^{\prime} 1\right)$ by one of the following conditions besides retaining the rest of the hypotheses:

$\left(\mathrm{e}^{\prime} 1\right)^{\prime}(X, d)$ is complete and one of $f$ and $g$ is onto,

$\left(\mathrm{e}^{\prime} 1\right)^{\prime \prime}(X, d)$ is complete and has a closed subspace $Y$ with $f(X) \subseteq Y \subseteq$ $g(X)$.

Proof. If $\left(\mathrm{e}^{\prime} 1\right)^{\prime}$ holds, then either $f(X)=X$ or $g(X)=X$ so that either $f(X)$ or $g(X)$ is complete and hence assumption $\left(\mathrm{e}^{\prime}\right)$ is applicable.

If $\left(\mathrm{e}^{\prime} 1\right)^{\prime \prime}$ holds, then $Y$ is complete and hence assumption $\left(\mathrm{e}^{\prime}\right)$ is applicable.

As commutativity $\Rightarrow$ weak commutativity $\Rightarrow$ compatibility for a pair of mappings, therefore the following consequence of Theorem 3.1 (also of Theorem 3.2) trivially holds.

Corollary 3.2. Theorem 3.1 (also Theorem 3.2) remains true if we replace condition (e2) by one of the following conditions besides retaining the rest of the hypotheses:

$(\mathrm{e} 2)^{\prime}(f, g)$ is commuting pair,

$(\mathrm{e} 2)^{\prime \prime}(f, g)$ is weakly commuting pair.

In the following lines, we present the results regarding the uniqueness of a point of coincidence and common fixed point corresponding to Theorems 3.1 and 3.2 .

Theorem 3.3. In addition to the hypotheses (a)-(d) along with ( $\left.\mathrm{e}^{\prime}\right)$ and (f) of Theorem 3.1 (also of Theorem 3.2), suppose that the following condition holds:

$\left(\mathrm{u}_{0}\right)$ every pair of elements of $f(X)$ has a lower bound or an upper bound in $g(X)$.

Then $f$ and $g$ have a unique point of coincidence.

Remark 3.2. In Theorem 3.3, we can replace $\left(\mathrm{u}_{0}\right)$ by the following condition:

$\left(\mathrm{u}_{0}^{\prime}\right)$ for every $x, y \in X, \exists z \in X$ such that $f(x) \prec \succ g(z)$ and $f(y) \prec \succ g(z)$.

Indeed $\left(\mathrm{u}_{0}\right)$ and $\left(\mathrm{u}_{0}^{\prime}\right)$ are equivalent. The implication $\left(\mathrm{u}_{0}\right) \Rightarrow\left(\mathrm{u}_{0}^{\prime}\right)$ is trivial.

Conversely, if $\left(\mathrm{u}_{0}^{\prime}\right)$ holds, then we have the following possibilities:

(i) $f(x) \preceq g(z)$ and $f(y) \preceq g(z)$ so that $g(z) \in g(X)$ is an upper bound of $\{f x, f y\}$

(ii) $g(z) \preceq f(x)$ and $g(z) \preceq f(y)$ so that $g(z) \in g(X)$ is a lower bound of $\{f x, f y\}$

(iii) $f(x) \preceq g(z) \preceq f(y)$ so that $f(y) \in g(X)$ is an upper bound of $\{f x, f y\}$,

(iv) $f(y) \preceq g(z) \preceq f(x)$ so that $f(x) \in g(X)$ is an upper bound of $\{f x, f y\}$, and hence $\left(\mathrm{u}_{0}\right)$ holds. Thus $\left(\mathrm{u}_{0}\right) \Leftrightarrow\left(\mathrm{u}_{0}^{\prime}\right)$. 
Theorem 3.4. In addition to the hypotheses of Theorem 3.3, suppose that the following condition holds:

$\left(\mathrm{u}_{1}\right)$ one of $f$ and $g$ is one-one.

Then $f$ and $g$ have a unique coincidence point.

Theorem 3.5. In addition to the hypotheses of Theorem 3.3, suppose that the following condition holds:

$\left(\mathrm{u}_{2}\right)(f, g)$ is weakly compatible pair.

Then $f$ and $g$ have a unique common fixed point.

Theorem 3.6. In addition to the hypotheses (a)-(e) along with (f) of Theorem 3.1 (similarly Theorem 3.2), if the condition $\left(\mathrm{u}_{0}\right)$ (of Theorem 3.3) holds, then $f$ and $g$ have a unique common fixed point.

We skip the proofs of Theorems 3.3-3.6 as these are similar to the proofs of Theorems $5-8$ of [2].

\section{Corresponding fixed point theorems}

By particularizing $g=I$, the identity mapping on $X$, in Theorems 3.2 and 3.1 (together with Theorems 3.3-3.6), we respectively derive the following fixed point theorems.

Theorem 4.1. Let $(X, d, \preceq)$ be an ordered metric space and $f$ a self-mapping on $X$. Suppose that the following conditions hold:

(i) there exists a subset $Y$ of $X$ such that $f(X) \subseteq Y$ and $(Y, d)$ is complete,

(ii) $f$ is monotone,

(iii) either $f$ is continuous or $(Y, d, \preceq)$ has TCC property,

(iv) there exists $x_{0} \in X$ such that $x_{0} \prec \succ f\left(x_{0}\right)$,

(v) there exists $\alpha \in[0,1)$ such that $d(f x, f y) \leq \alpha d(x, y) \quad \forall x, y \in X$ with $x \prec \succ y$.

Then $f$ has a fixed point. Moreover, if in addition the following also holds:

(vi) every pair of elements of $f(X)$ has a lower bound or an upper bound, then $f$ has a unique fixed point.

Remark 4.1. Notice that Theorem 4.1 improves Theorem 1.1 (i.e., the main result of Ran and Reurings [35]) and Theorem 1.2 (i.e., the main result of Nieto and Rodríguez-López [32]) in the following respects:

- In the context of hypothesis (i), the completeness of $X$ is not necessary. Alternately, it can be replaced by the completeness of $Y$, where $f(X) \subseteq Y \subseteq X$.

- In the context of hypothesis (vi), the requirement of a lower bound or an upper bound is not required on whole of $X$ but it suffices to take the same merely on the subset $f(X)$.

- The assumption (vi) is unnecessary for the existence part and it is merely utilized to establish the uniqueness of fixed point.

Theorem 4.2. Let $(X, d, \preceq)$ be an ordered metric space and $f$ a self-mapping on $X$. Suppose that the following conditions hold: 
(i) there exists a subset $Y$ of $X$ such that $f(X) \subseteq Y$ and $(Y, d)$ is complete,

(ii) $f$ is monotone,

(iii) either $f$ is continuous or $(Y, d, \preceq)$ has TCC property,

(iv) there exists $x_{0} \in X$ such that $x_{0} \prec \succ f\left(x_{0}\right)$,

(v) there exists $\varphi \in \Omega$ such that $d(f x, f y) \leq \varphi(d(x, y)) \quad \forall x, y \in X$ with $x \prec \succ y$,

(vi) $(f X, \preceq)$ is sequentially chainable.

Then $f$ has a fixed point. Moreover, if in addition the following also holds:

(vii) every pair of elements of $f(X)$ has a lower bound or an upper bound, then $f$ has a unique fixed point.

Remark 4.2. Indeed, Theorem 4.2 is an extension of Theorem 4.1 (and hence that of Theorems 1.1 and 1.2) to Boyd-Wong type nonlinear contractions. Here, it can be observed that this extension is not straight forward due to additional requirement: " $(f X, \preceq)$ is sequentially chainable". But on restricting to linear contractions (from nonlinear contractions), we deduce well-known fixed point theorems of Ran and Reurings and Nieto and Rodríguez-López (i.e., Theorems 1.1 and 1.2 ).

\section{Examples}

In this section, we furnish some examples establishing the genuineness of our main results.

Example 5.1. Consider $X=[0, \infty)$ equipped with usual metric $d$ and usual partial order $\preceq$. Then $(X, d)$ is a complete metric space. Define a mapping $f: X \rightarrow X$ by $f(x)=\frac{x}{x+1} \forall x \in X$. Then $f$ is increasing. Define $\varphi:[0, \infty) \rightarrow$ $[0, \infty)$ by $\varphi(t)=\frac{t}{1+t} \forall t \in[0, \infty)$, then $\varphi \in \Omega$. Now, for all $x, y \in X$ with $x \succeq y$, we have

$$
\begin{aligned}
d(f x, f y) & =\frac{x}{x+1}-\frac{y}{y+1}=\frac{x-y}{1+x+y+x y} \\
& \leq \frac{x-y}{1+(x-y)}=\frac{d(x, y)}{1+d(x, y)}=\varphi(d(x, y))
\end{aligned}
$$

so that $f$ and $\varphi$ satisfy assumption (v) of Theorem 4.1. Observe that all the other conditions of Theorem 4.1 are also satisfied. Therefore, $f$ has a unique fixed point (namely: $x=0$ ).

Notice that $f$ is not a linear contraction. To substantiate this, choose $x=0$ and $y=\epsilon$, where $\epsilon$ is arbitrarily small but positive. If we take a constant $\alpha$ such that $d(f x, f y) \leq \alpha d(x, y)$, then $\alpha \geq \frac{1}{1+\epsilon}$, which amounts to say that $\alpha \geq 1$ so that $\alpha \notin[0,1)$. Henceforth, $f$ is not a linear contraction. Thus, Example 5.1 establishes the utility of Theorem 4.1 over well known fixed point theorems of Ran and Reurings and Nieto and Rodríguez-López (i.e., Theorems 1.1 and $1.2)$. 
Example 5.2. Consider $X=\mathbb{R}$ equipped with usual metric and usual partial order. Define $f, g: X \rightarrow X$ by $f(x)=5$ and $g(x)=x^{2}-4 \forall x \in X$. Then $f$ is $g$-monotone. Let $\varphi \in \Omega$ be arbitrary. Now, for $x, y \in X$ with $g(x) \preceq g(y)$, we have

$$
d(f x, f y)=|5-5|=0 \leq \varphi\left(\left|x^{2}-y^{2}\right|\right)=\varphi(d(g x, g y))
$$

Thus $f, g$ and $\varphi$ satisfy the assumption (d) of Theorem 3.1. Also, the pair $(f, g)$ is not compatible and hence (e) does not hold. But the subspace $g(X)=$ $[-4, \infty)$ is complete and $f$ and $g$ are continuous, i.e., all the conditions mentioned in $\left(\mathrm{e}^{\prime}\right)$ are satisfied. Hence by Theorem 3.1, $f$ and $g$ have a coincidence point in $X$. Further, in this example $\left(\mathrm{u}_{0}\right)$ holds and henceforth, in view of Theorem 3.3, $f$ and $g$ have a unique point of coincidence (namely: $\bar{x}=5$ ). Notice that neither $f$ nor $g$ is one-one, i.e., $\left(u_{1}\right)$ does not hold and hence, we can not apply Theorem 3.4, which guarantees the uniqueness of coincidence point. Observe that there are two coincidence points (namely: $x=3$ and $x=-3$ ). Also, the pair $(f, g)$ is not weakly compatible, i.e., $\left(\mathrm{u}_{2}\right)$ does not hold and hence, we can not apply Theorem 3.5, which ensures the uniqueness of common fixed point. Notice that there is no common fixed point of $f$ and $g$.

Example 5.3. Let $X=\mathbb{R}$. On $X$, consider usual metric $d$ and partial order $\preceq$ defined by $x \preceq y \Leftrightarrow x \leq y$ and $x y \geq 0$. Then $(X, d)$ is a complete metric space. Define $f, g: X \rightarrow X$ by $f(x)=\frac{x^{2}}{3}$ and $g(x)=-x^{2} \forall x \in X$. Then $f$ is $g$-decreasing. Define $\varphi:[0, \infty) \rightarrow[0, \infty)$ by $\varphi(t)=\frac{2 t}{3} \forall t \in[0, \infty)$, then $\varphi \in \Omega$. Now, for $x, y \in X$ with $g(x) \preceq g(y)$, we have

$d(f x, f y)=\left|\frac{x^{2}}{3}-\frac{y^{2}}{3}\right|=\frac{1}{3}\left|x^{2}-y^{2}\right|=\frac{1}{3} d(g x, g y)<\frac{2}{3} d(g x, g y)=\varphi(d(g x, g y))$.

Therefore, $f, g$ and $\varphi$ satisfy assumption (d) of Theorem 3.1. By a routine calculation, one can also verify all the conditions mentioned in (e) (of Theorem 3.1). Thus, all the conditions of Theorem 3.1 are satisfied and $f$ and $g$ have a coincidence point in $X$. Moreover, the condition $\left(\mathrm{u}_{0}\right)$ also holds and therefore, in view of Theorem 3.6, $f$ and $g$ have a unique common fixed point (namely: $x=0)$.

\section{Application}

In this section, as an application of Theorem 4.2, we prove an existence and uniqueness of solution of the following first order periodic boundary value problem which is essentially inspired by [32].

$$
\left\{\begin{array}{l}
u^{\prime}(t)=f(t, u(t)), \quad t \in I=[0, T] \\
u(0)=u(T),
\end{array}\right.
$$

where $T>0$ and $f: I \times \mathbb{R} \rightarrow \mathbb{R}$ is a continuous function.

Let $\mathcal{C}(I)$ denote the space of all continuous functions defined on $I$. Now, we need to recall the following definitions: 
Definition 6.1 ([32]). A function $\alpha \in \mathcal{C}^{1}(I)$ is called a lower solution of (21), if

$$
\left\{\begin{array}{l}
\alpha^{\prime}(t) \leq f(t, \alpha(t)), \quad t \in I \\
\alpha(0) \leq \alpha(T)
\end{array}\right.
$$

Definition 6.2 ([32]). A function $\alpha \in \mathcal{C}^{1}(I)$ is called an upper solution of (21), if

$$
\left\{\begin{array}{l}
\alpha^{\prime}(t) \geq f(t, \alpha(t)), \quad t \in I \\
\alpha(0) \geq \alpha(T)
\end{array}\right.
$$

Let $\mathfrak{F}$ denote the family of functions $\phi:[0, \infty] \rightarrow[0, \infty]$ satisfying the following conditions:

(i) $\phi$ is continuous and increasing,

(ii) $\phi(t)<t$ for each $t>0$.

Typical examples of $\mathfrak{F}$ are $\phi(t)=\alpha t, 0 \leq \alpha<1, \phi(t)=\frac{t}{1+t}$ and $\phi(t)=$ $\ln (1+t)$. Also, clearly $\mathfrak{F} \subset \Omega$.

Now, we prove the following result regarding the existence and uniqueness of the solution of the periodic boundary value problem described by (21) in the presence of a lower solution or an upper solution.

Theorem 6.1. In addition to the problem described by (21), suppose that there exist $\lambda>0$ and $\phi \in \mathfrak{F}$ such that for all $x, y \in \mathbb{R}$ with $x \leq y$

$$
-\lambda \phi(y-x) \leq f(t, y)+\lambda y-[f(t, x)+\lambda x] \leq 0 .
$$

Then the existence of a lower solution or an upper solution of problem (21) ensures the existence and uniqueness of the solution of the periodic boundary value problem described by (21).

Proof. The problem (21) can be rewritten as:

$$
\left\{\begin{array}{l}
u^{\prime}(t)+\lambda u(t)=f(t, u(t))+\lambda u(t) \quad \forall t \in I \\
u(0)=u(T)
\end{array}\right.
$$

Notice that the problem (23) is equivalent to the integral equation

$$
u(t)=\int_{0}^{T} G(t, \xi)[f(\xi, u(\xi))+\lambda u(\xi)] d \xi,
$$

where the Green function $G(t, \xi)$ is given by

$$
G(t, \xi)= \begin{cases}\frac{e^{\lambda(T+\xi-t)}}{e^{\lambda T}-1} & 0 \leq \xi<t \leq T \\ \frac{e^{\lambda(\xi-t)}}{e^{\lambda T}-1} & 0 \leq t<\xi \leq T .\end{cases}
$$

Define a function $\mathcal{A}: \mathcal{C}(I) \rightarrow \mathcal{C}(I)$ by

$$
(\mathcal{A} u)(t)=\int_{0}^{T} G(t, \xi)[f(\xi, u(\xi))+\lambda u(\xi)] d \xi \forall t \in I .
$$


Evidently, if $u \in \mathcal{C}(I)$ is a fixed point of $\mathcal{A}$, then $u \in \mathcal{C}^{1}(I)$ is a solution of $(24)$ and hence of $(21)$.

On $\mathcal{C}(I)$, define a metric $d$ given by:

$$
d(u, v)=\sup _{t \in I}|u(t)-v(t)| \quad \forall u, v \in \mathcal{C}(I) .
$$

Also, on $\mathcal{C}(I)$, define a partial order $\preceq$ given by:

$$
u, v \in \mathcal{C}(I) ; u \preceq v \Longleftrightarrow u(t) \leq v(t) \forall t \in I .
$$

Now, we check that all the conditions of Theorem 4.2 are satisfied for $Y=$ $X=\mathcal{C}(I)$.

(i) Clearly, $(\mathcal{C}(I), d)$ is a complete metric space.

(ii) Take $u, v \in \mathcal{C}(I)$ such that $u \preceq v$, then by (22), we obtain

$$
f(t, u(t))+\lambda u(t) \geq f(t, v(t))+\lambda v(t) \forall t \in I .
$$

On using (24), (25) and the fact that $G(t, \xi)>0$ for $(t, \xi) \in I \times I$, we get

$$
\begin{aligned}
(\mathcal{A} u)(t) & =\int_{0}^{T} G(t, \xi)[f(\xi, u(\xi))+\lambda u(\xi)] d \xi \\
& \geq \int_{0}^{T} G(t, \xi)[f(\xi, v(\xi))+\lambda v(\xi)] d \xi \\
& =(\mathcal{A} v)(t) \quad \forall t \in I,
\end{aligned}
$$

which implies that $\mathcal{A}(u) \succeq \mathcal{A}(v)$ so that $\mathcal{A}$ is decreasing.

(iii) Take a sequence $\left\{u_{n}\right\} \subset \mathcal{C}(I)$ such that $u_{n} \uparrow u \in \mathcal{C}(I)$. Then for each $t \in I,\left\{u_{n}(t)\right\}$ is a sequence in $\mathbb{R}$ converging to $u(t)$. Hence, $\left\{u_{n}(t)\right\}$ has a monotone subsequence $\left\{u_{n_{k}}(t)\right\}$. Therefore, for all $k \in \mathbb{N}_{0}$ and for all $t \in I$, we have

$$
\begin{array}{ll}
u_{n_{k}}(t) \leq u(t) & \text { if }\left\{u_{n_{k}}(t)\right\} \text { is increasing } \\
u_{n_{k}}(t) \geq u(t) & \text { if }\left\{u_{n_{k}}(t)\right\} \text { is decreasing, }
\end{array}
$$

which implies that $u_{n_{k}} \prec \succ u \forall k \in \mathbb{N}_{0}$ so that $(\mathcal{C}(I), d, \preceq)$ has $T C C$ property.

(iv) Let $\alpha \in \mathcal{C}^{1}(I)$ be a lower solution of (21), then we have

$$
\alpha^{\prime}(t)+\lambda \alpha(t) \leq f(t, \alpha(t))+\lambda \alpha(t) \forall t \in I .
$$

Multiplying on both the sides by $e^{\lambda t}$, we get

$$
\left(\alpha(t) e^{\lambda t}\right)^{\prime} \leq[f(t, \alpha(t))+\lambda \alpha(t)] e^{\lambda t} \forall t \in I,
$$

which implies that

$$
\alpha(t) e^{\lambda t} \leq \alpha(0)+\int_{0}^{t}[f(\xi, \alpha(\xi))+\lambda \alpha(\xi)] e^{\lambda \xi} d \xi \quad \forall t \in I .
$$

As $\alpha(0) \leq \alpha(T)$, we get

$$
\alpha(0) e^{\lambda T} \leq \alpha(T) e^{\lambda T} \leq \alpha(0)+\int_{0}^{T}[f(\xi, \alpha(\xi))+\lambda \alpha(\xi)] e^{\lambda \xi} d \xi
$$


so that

$$
\alpha(0) \leq \int_{0}^{T} \frac{e^{\lambda \xi}}{e^{\lambda T}-1}[f(\xi, \alpha(\xi))+\lambda \alpha(\xi)] d \xi .
$$

On using (26) and (27), we obtain

$$
\begin{aligned}
\alpha(t) e^{\lambda t} & \leq \int_{0}^{T} \frac{e^{\lambda \xi}}{e^{\lambda T}-1}[f(\xi, \alpha(\xi))+\lambda \alpha(\xi)] d \xi+\int_{0}^{t} e^{\lambda \xi}[f(\xi, \alpha(\xi))+\lambda \alpha(\xi)] d \xi \\
& =\int_{0}^{t} \frac{e^{\lambda(T+\xi)}}{e^{\lambda T}-1}[f(\xi, \alpha(\xi))+\lambda \alpha(\xi)] d \xi+\int_{t}^{T} \frac{e^{\lambda \xi}}{e^{\lambda T}-1}[f(\xi, \alpha(\xi))+\lambda \alpha(\xi)] d \xi
\end{aligned}
$$

so that

$$
\begin{aligned}
\alpha(t) & \leq \int_{0}^{t} \frac{e^{\lambda(T+\xi-t)}}{e^{\lambda T}-1}[f(\xi, \alpha(\xi))+\lambda \alpha(\xi)] d \xi+\int_{t}^{T} \frac{e^{\lambda(\xi-t)}}{e^{\lambda T}-1}[f(\xi, \alpha(\xi))+\lambda \alpha(\xi)] d \xi \\
& =\int_{0}^{T} G(t, \xi)[f(\xi, \alpha(\xi))+\lambda \alpha(\xi)] d \xi \\
& =(\mathcal{A} \alpha)(t)
\end{aligned}
$$

for all $t \in I$, which implies that $\alpha \preceq \mathcal{A}(\alpha)$. Otherwise, if $\alpha \in \mathcal{C}^{1}(I)$ is an upper solution of (21), then in the similar manner, we get $\alpha \succeq \mathcal{A}(\alpha)$. Hence, in both the cases, we have $\alpha \prec \succ \mathcal{A}(\alpha)$, for some lower or upper solution $\alpha$.

(v) Take $u, v \in \mathcal{C}(I)$ such that $u \preceq v$, we have

$$
\begin{aligned}
d(\mathcal{A} u, \mathcal{A} v) & =\sup _{t \in I}|(\mathcal{A} u)(t)-(\mathcal{A} v)(t)|=\sup _{t \in I}((\mathcal{A} u)(t)-(\mathcal{A} v)(t)) \\
& \leq \sup _{t \in I} \int_{0}^{T} G(t, \xi)[f(\xi, u(\xi))+\lambda u(\xi)-f(\xi, v(\xi))-\lambda v(\xi)] d \xi \\
& \leq \sup _{t \in I} \int_{0}^{T} G(t, \xi) \lambda \phi(v(\xi)-u(\xi)) d \xi .
\end{aligned}
$$

Given that $\phi$ is increasing on $[0, \infty)$ and $u \preceq v$, which implies that $\phi(v(\xi)-$ $u(\xi)) \leq \phi(d(u, v))$. Hence, $(28)$ reduces to

$$
\begin{aligned}
d(\mathcal{A} u, \mathcal{A} v) & \leq \lambda \phi(d(u, v)) \sup _{t \in I} \int_{0}^{T} G(t, \xi) d \xi \\
& =\lambda \phi(d(u, v)) \sup _{t \in I} \frac{1}{e^{\lambda T}-1}\left(\left[\frac{1}{\lambda} e^{\lambda(T+\xi-t)}\right]_{0}^{t}+\left[\frac{1}{\lambda} e^{\lambda(\xi-t)}\right]_{t}^{T}\right) \\
& =\lambda \phi(d(u, v)) \frac{1}{\lambda\left(e^{\lambda T}-1\right)}\left(e^{\lambda T}-1\right) \\
& =\phi(d(u, v))
\end{aligned}
$$

so that

$$
d(\mathcal{A} u, \mathcal{A} v) \leq \phi(d(u, v)) \forall u, v \in \mathcal{C}(I) \text { such that } u \preceq v,
$$

where $\phi \in \mathfrak{F} \subset \Omega$.

(vi) It is easy to check that $(\mathcal{A C}(I), \preceq)$ is sequentially chainable. 
Hence, the conditions (i)-(vi) of Theorem 4.2 are satisfied for $Y=X=\mathcal{C}(I)$ consequently $\mathcal{A}$ has a fixed point.

Choose arbitrary $u, v \in \mathcal{C}(I)$, then $w:=\max \{\mathcal{A} u, \mathcal{A} v\} \in \mathcal{C}(I)$, which yields that $w$ is an upper bound of $\{\mathcal{A} u, \mathcal{A} v\}$. Thus, by Theorem $4.2, \mathcal{A}$ has a unique fixed point, which is, indeed, a unique solution of problem (21).

\section{References}

[1] R. P. Agarwal, M. A. El-Gebeily, and D. O'Regan, Generalized contractions in partially ordered metric spaces, Appl. Anal. 87 (2008), no. 1, 109-116.

[2] A. Alam, A. R. Khan, and M. Imdad, Some coincidence theorems for generalized nonlinear contractions in ordered metric spaces with applications, Fixed Point Theory Appl. 2014 (2014), 216, 30 pp.

[3] I. Altun and H. Simsek, Some fixed point theorems on ordered metric spaces and application, Fixed Point Theory Appl. 2010 (2010), 621469, 17 pp.

[4] H. Amann, Order structures and fixed points, Bochum: Mimeographed lecture notes, Ruhr-Universität, 1977.

[5] A. Amini-Harandi and H. Emami, A fixed point theorem for contraction type maps in partially ordered metric spaces and application to ordinary differential equations, Nonlinear Anal. 72 (2010), no. 5, 2238-2242.

[6] A. Björner, Order-reversing maps and unique fixed points in complete lattices, Algebra Universalis 12 (1981), no. 3, 402-403.

[7] D. W. Boyd and J. S. W. Wong, On nonlinear contractions, Proc. Amer. Math. Soc. 20 (1969), 458-464.

[8] J. Caballero, J. Harjani, and K. Sadarangani, Contractive-like mapping principles in ordered metric spaces and application to ordinary differential equations, Fixed Point Theory Appl. 2010 (2010), Art. ID 916064, 14 pp.

[9] S. Carl, A monotone iterative scheme for nonlinear reaction-diffusion systems having nonmonotone reaction terms, J. Math. Anal. Appl. 134 (1988), no. 1, 81-93.

[10] — An enclosing theorem and a monotone iterative scheme for elliptic systems having nonmonotone nonlinearities, Z. Angew. Math. Mech. 70 (1990), no. 8, 309-313.

[11] S. Carl and S. Heikkilä, Fixed Point Theory in Ordered Sets and Applications: from differential and integral equations to game theory, Springer, New York, 2011.

[12] L. Ćirić, N. Cakic, M. Rajovic, and J. S. Ume, Monotone generalized nonlinear contractions in partially ordered metric spaces, Fixed Point Theory Appl. 2008 (2008), Art. ID 131294, $11 \mathrm{pp.}$

[13] P. Cousot and R. Cousot, Constructive versions of Tarski's fixed point theorems, Pacific J. Math. 82 (1979), no. 1, 43-57.

[14] R. DeMarr, Common fixed points for isotone mappings, Colloq. Math. 13 (1964), 45-48.

[15] R. H. Haghi, S. Rezapour, and N. Shahzad, Some fixed point generalizations are not real generalizations, Nonlinear Anal. 74 (2011), no. 5, 1799-1803.

[16] J. Harjani and K. Sadarangani, Fixed point theorems for weakly contractive mappings in partially ordered sets, Nonlinear Anal. 71 (2009), no. 7-8, 3403-3410.

[17] - Generalized contractions in partially ordered metric spaces and applications to ordinary differential equations, Nonlinear Anal. 72 (2010), no. 3-4, 1188-1197.

[18] S. Heikkilä and V. Lakshmikantham, Monotone Iterative Techniques for Discontinuous Nonlinear Differential Equations, Marcel Dekker, Inc., New York, 1994.

[19] J. Jachymski, Equivalent conditions for generalized contractions on (ordered) metric spaces, Nonlinear Anal. 74 (2011), no. 3, 768-774.

[20] M. Jleli, V. C. Rajic, B. Samet, and C. Vetro, Fixed point theorems on ordered metric spaces and applications to nonlinear elastic beam equations, J. Fixed Point Theory Appl. 12 (2012), no. 1-2, 175-192. 
[21] N. Jotic, Some fixed point theorems in metric spaces, Indian J. Pure Appl. Math. 26 (1995), no. 10, 947-952.

[22] G. Jungck, Commuting maps and fixed points, Amer. Math. Monthly 83 (1976), no. 4, 261-263.

[23] Compatible mappings and common fixed points, Int. J. Math. Math. Sci. 9 (1986), no. 4, 771-779.

[24] - Common fixed points for noncontinuous nonself maps on non-metric spaces, Far East J. Math. Sci. 4 (1996), no. 2, 199-215.

[25] D. Kurepa, Fixpoints of decreasing mappings of ordered sets, Publ. Inst. Math. (N.S.) 18(32) (1975), 111-116.

[26] G. S. Ladde, V. Lakshmikantham, and A. S. Vatsala, Monotone Iterative Techniques for Nonlinear Differential Equations, Pitman, Boston, 1985.

[27] V. Lakshmikantham and L. Ćirić, Coupled fixed point theorems for nonlinear contractions in partially ordered metric spaces, Nonlinear Anal. 70 (2009), no. 12, 4341-4349.

[28] S. Lipschutz, Schaum's Outlines of Theory and Problems of Set Theory and Related Topics, McGraw-Hill, 1964.

[29] A. Mukherjea, Contractions and completely continuous mappings, Nonlinear Anal. 1 (1977), no. 3, 235-247.

[30] H. K. Nashine and I. Altun, A common fixed point theorem on ordered metric spaces, Bull. Iranian Math. Soc. 38 (2012), no. 4, 925-934.

[31] J. J. Nieto and R. Rodríguez-López, Contractive mapping theorems in partially ordered sets and applications to ordinary differential equations, Order 22 (2005), no. 3, 223-239.

[32] _ Existence and uniqueness of fixed point in partially ordered sets and applications to ordinary differential equation, Acta Math. Sin. (Engl. Ser.) 23 (2007), no. 12, 22052212.

[33] D. O'Regan and A. Petruşel, Fixed point theorems for generalized contractions in ordered metric spaces, J. Math. Anal. Appl. 341 (2008), no. 2, 1241-1252.

[34] A. Petruşel and I. A. Rus, Fixed point theorems in ordered L-spaces, Proc. Amer. Math. Soc. 134 (2006), no. 2, 411-418.

[35] A. C. M. Ran and M. C. B. Reurings, A fixed point theorem in partially ordered sets and some applications to matrix equations, Proc. Amer. Math. Soc. 132 (2004), no. 5, 1435-1443.

[36] K. P. R. Sastry and I. S. R. Krishna Murthy, Common fixed points of two partially commuting tangential selfmaps on a metric space, J. Math. Anal. Appl. 250 (2000), no. 2, 731-734.

[37] S. Sessa, On a weak commutativity condition of mappings in fixed point considerations, Publ. Inst. Math. (Beograd) (N.S.) 32(46) (1982), 149-153.

[38] A. Tarski, A lattice-theoretical fixpoint theorem and its applications, Pacific J. Math. 5 (1955), 285-309.

[39] M. Turinici, Fixed points for monotone iteratively local contractions, Demonstr. Math. 19 (1986), no. 1, 171-180.

[40] J. S. W. Wong, Common fixed points of commuting monotone mappings, Canad. J. Math. 19 (1967), 617-620.

[41] J. Wu and Y. Liu, Fixed point theorems for monotone operators and applications to nonlinear elliptic problems, Fixed Point Theory Appl. 2013 (2013), 134, 14 pp.

Aftab Alam

Department of Mathematics

Aligarh Muslim University

Aligarh 202002, Uttar Pradesh, India

E-mail address: aafu.amu@gmail.com 
MOHAMMAD IMDAD

Department of Mathematics

Aligarh Muslim University

Aligarh 202002, Uttar Pradesh, India

E-mail address: mhimdad@yahoo.co.in 